\title{
Characterization of Two-Dimensional Finite-Aperture Wire Grid Polarizers by a Spectral-Domain Technique
}

\author{
Michael A. Jensen \\ jensen@byu.edu \\ Gregory P. Nordin \\ nordin@byu.edu
}

Follow this and additional works at: https://scholarsarchive.byu.edu/facpub

Part of the Electrical and Computer Engineering Commons

\section{Original Publication Citation}

M. A. Jensen and G. P. Nordin, "Characterization of Two-Dimensional Finite-Aperture Wire Grid Polarizers by a Spectral-Domain Technique," Appl. Opt. 4(26) pp. 4738-4745 (21)

\section{BYU ScholarsArchive Citation}

Jensen, Michael A. and Nordin, Gregory P., "Characterization of Two-Dimensional Finite-Aperture Wire Grid Polarizers by a Spectral-Domain Technique" (2001). Faculty Publications. 1083.

https://scholarsarchive.byu.edu/facpub/1083 


\title{
Characterization of two-dimensional finite-aperture wire grid polarizers by a spectral-domain technique
}

\author{
Michael A. Jensen and Gregory P. Nordin
}

\begin{abstract}
We investigate the transmission characteristics of perfectly conducting two-dimensional wire grid polarizers fabricated in finite and infinite apertures using a rigorous spectral-domain mode-matching method. Specifically, the transmission coefficient for both transverse-electric and transverse-magnetic polarizations, extinction ratio, and diffraction pattern are characterized for a wide variety of geometric and material parameters including aperture dimension, conducting wire fill factor, wire spacing, polarizer thickness, material dielectric constants, and incident wave arrival angle. The results indicate that the transmission behavior is largely insensitive to aperture dimension. (c) 2001 Optical Society of America

OCIS codes: $\quad 050.0050,050.1970,230.0230,230.3990$.
\end{abstract}

\section{Introduction}

It has long been recognized that wire grid polarizers are an effective option for discriminating between orthogonal linear polarization states at infrared wavelengths..$^{1,2}$ Such polarizers are typically fabricated when conducting wires are placed within an aperture formed in a larger opaque screen. When the aperture is electrically large, the device behavior is similar to that of a polarizer of infinite extent, and its performance can be accurately characterized with simulation approaches such as rigorous coupled-wave analysis. ${ }^{3,4}$ However, with the emergence of new fabrication technologies have come a host of applications that require implementation of small-aperture polarizers ${ }^{5}$ and arrays of micropolarizers, ${ }^{6,7}$ motivating the development of devices with electrically small apertures. In this case, alternative simulation techniques that model the finite-aperture extent must be explored for device characterization.

A variety of research has appeared on the subject of modeling finite-sized diffractive optical devices. Most of these studies have focused on grating structures, diffractive lenses, and other aperiodic geometries and

M. A. Jensen (jensen@ee.byu.edu) is with the Department of Electrical and Computer Engineering, 459 Clyde Building, Brigham Young University, Provo, Utah 84602. G. P. Nordin (nordin@ece.uah.edu) is with the Department of Electrical and Computer Engineering, University of Alabama, Huntsville, Huntsville, Alabama 35899.

Received 22 March 2001; revised manuscript received 15 June 2001.

0003-6935/01/264738-08\$15.00/0

(C) 2001 Optical Society of America exploit simplifying assumptions based on Kirchhoff's scalar solutions. ${ }^{8}$ Other studies have used computational methods such as the boundary element method $^{9-11}$ and method of moments ${ }^{12,13}$ to more accurately describe the device behavior. More recently, we used a finite-difference time-domain implementation to characterize the behavior of polarizers with finite-extent-aperture size in both transverse dimensions. ${ }^{14}$ Although this research has brought forth useful information for the characterization of these polarizer structures, the computational complexity and material modeling constraints of the method have precluded exhaustive exploration of polarizer behavior as a function of a broad range of geometric and material parameters. This fact motivates investigation of these geometries with simpler and less computationally intensive analytical techniques.

In response to this need, we present in this paper a detailed characterization of perfectly conducting wire grid polarizers placed in finite apertures by a spectral-domain mode-matching technique. ${ }^{15-19}$ The resulting solution allows highly accurate simulations of two-dimensional polarizer structures for both transverse-electric (TE) and transversemagnetic (TM) illuminations. This approach is advantageous as it allows a relatively straightforward examination of a wide variety of configurations, thereby facilitating detailed studies that illustrate the effect of key geometric parameters on the polarizer performance.

In this paper we provide a brief yet informative summary of the computational method and discuss computational issues associated with its implementation in Section 2. This discussion includes an extension of 


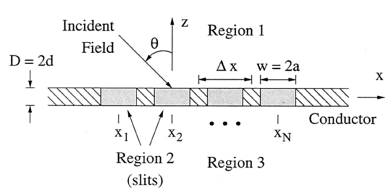

Fig. 1. Geometry for a plane wave incident upon the finiteaperture wire polarizer.

the technique that uses Floquet analysis to accommodate infinitely long polarizers. In Section 3 we define the polarizer parameters of interest and study the influence of aperture size, wire properties (fill factor, slit density, and wire thickness), material characteristics, and illuminating field incidence angle on the transmissivity and diffraction pattern of the polarizer. Finally, we provide conclusions in Section 4.

\section{Analysis Methodology}

The problem under consideration is that of a plane wave impinging on a finite-sized aperture located in an otherwise infinite conducting plane of thickness $D=$ $2 d$. As indicated in Fig. 1, the wave vector of the incident plane wave lies in the $x-z$ plane and makes an angle $\theta$ with the $z$ axis. The aperture is divided into $N$ slits, each of width $w=2 a$, with the $n$th slit centered at $x=x_{n}$. The center-to-center spacing of the conducting regions between the slits is denoted as $\Delta x$. The wave number, permittivity, and permeability in each region are expressed as $k_{j}, \epsilon_{j}$, and $\mu_{j}$, respectively, where $j$ indicates the region number 1,2 , or 3 as defined in Fig. 1.

The structure depicted in Fig. 1 can be analyzed with a spectral-domain mode-matching technique. This approach has the limitation of modeling only two-dimensional structures and therefore cannot predict the influence of the finite-aperture size in the $y$ dimension. In addition, it cannot accommodate finite conductivity for the materials that comprise the wires. However, in contrast to more sophisticated computational methodologies, this technique has the advantage of relatively simple implementation and low computational requirements. This latter features makes it possible to explore performance trends as a function of a wide variety of geometric parameters. Also, the method allows simple exploration of the effect of different medium characteristics (in regions 1-3) on the polarizer behavior. This is particularly relevant because typical polarizers are fabricated on a dielectric substrate. Finally, it should be emphasized that the results observed with the two-dimensional model provide invaluable physical insight into the expected behavior of threedimensional structures with finite-aperture size in both the $x$ and $y$ dimensions.

\section{A. Field Solutions}

The starting point in the analysis is the proper expression of the fields in the three regions in Fig. 1. For a TE-polarized wave, the fields in region 1 can be repre- sented as the sum of incident, reflected, and scattered components that can each be expressed as

$$
\begin{aligned}
E_{y}{ }^{i}(x, z)= & \exp \left[i k_{x} x-i k_{z}(z-d)\right] \\
E_{y}{ }^{r}(x, z)= & -\exp \left[i k_{x} x+i k_{z}(z-d)\right] \\
E_{y}{ }^{s}(x, z)= & \frac{1}{2 \pi} \int_{-\infty}^{\infty} \tilde{E}_{y}^{s}(\xi) \exp \left[-i \xi x+i\left(k_{1}{ }^{2}-\xi^{2}\right)^{1 / 2}\right. \\
& \times(z-d)] \mathrm{d} \xi \\
\tilde{E}_{y}{ }^{s}(\xi)= & \int_{-\infty}^{\infty} E_{y}^{s}(x, d) \exp (i \xi x) \mathrm{d} x
\end{aligned}
$$

where $k_{x}=k_{1} \sin \theta$ and $k_{z}=k_{1} \cos \theta$. The spectral representation in Eq. (3) for the scattered field facilitates later enforcement of continuity conditions. In a similar fashion, the field transmitted into region 3 can be expressed as

$$
\begin{aligned}
E_{y}{ }^{t}(x, z)= & \frac{1}{2 \pi} \int_{-\infty}^{\infty} \tilde{E}_{y}{ }^{t}(\xi) \\
& \times \exp \left[-i \xi x-i\left(k_{3}{ }^{2}-\xi^{2}\right)^{1 / 2}(z+d)\right] \mathrm{d} \xi \\
\tilde{E}_{y}{ }^{t}(\xi)= & \int_{-\infty}^{\infty} E_{y}^{t}(x,-d) \exp (i \xi x) \mathrm{d} x
\end{aligned}
$$

Finally, the fields in the $n$th slit in region 2 can be expressed by use of modes that satisfy the boundary conditions on the slit sidewalls. This results in the summation

$$
\begin{aligned}
E_{y}{ }^{n}(x, z)= & \sum_{m=1}^{\infty}\left(b_{m}{ }^{n} \cos \zeta_{m} z+c_{m}{ }^{n} \sin \zeta_{m} z\right) \\
& \times \sin \left[a_{m}\left(x-x_{n}+a\right)\right],
\end{aligned}
$$

where $a_{m}=m \pi / 2 a$ and $\zeta_{m}=\left(k_{2}{ }^{2}-a_{m}{ }^{2}\right)^{1 / 2}$.

Using these field representations, we must now enforce continuity of tangential fields at $z= \pm d$ and solve for unknown quantities. For convenience, we define the functions

$$
G_{1,2}(m, \xi)=\frac{(-1)^{m} \exp (\mp i \xi a)-\exp ( \pm i \xi a)}{\xi^{2}-a_{m}^{2}},
$$

where the upper and lower signs are for $G_{1}$ and $G_{2}$, respectively. Enforcing continuity of $E_{y}$, we obtain the expression

$$
\begin{aligned}
\tilde{E}_{y}^{s, t}(\xi)= & \sum_{n=1}^{N} \sum_{m=1}^{\infty}\left(b_{m}{ }^{n} \cos \zeta_{m} d\right. \\
& \left. \pm c_{m}{ }^{n} \sin \zeta_{m} d\right) a_{m} G_{2}(m, \xi) \exp \left(i \xi x_{n}\right),
\end{aligned}
$$

where here, as well as in other equations in this paper, the upper and lower signs are used for scattered and transmitted fields, respectively. Now, using the relation $H_{x}=(i / \omega \mu) \partial E_{y} / \partial z$, we can enforce continuity of the magnetic fields over each slit. If we then approx- 
imate the infinite series in Eq. (9) using the first $M$ terms and order the unknowns $b_{m}{ }^{n}$ and $c_{m}{ }^{n}$ into column vectors $B$ and $C$, we obtain the block matrix equation

$$
\left[\begin{array}{rr}
\Psi_{1 B} & \Psi_{1 C} \\
\Psi_{3 B} & -\Psi_{3 C}
\end{array}\right]\left[\begin{array}{l}
B \\
C
\end{array}\right]=\left[\begin{array}{l}
\Gamma \\
0
\end{array}\right]
$$

where

$$
\Psi_{l B, r s}=\zeta_{p} a \frac{\mu_{l}}{\mu_{2}} \sin \left(\zeta_{p} d\right) \delta_{r s}+\frac{i a_{m} a_{p}}{2 \pi} \cos \left(\zeta_{m} d\right) I_{p m q n}\left(k_{l}\right)
$$

$$
\begin{aligned}
\Psi_{l C, r s}= & -\zeta_{p} a \frac{\mu_{l}}{\mu_{2}} \cos \left(\zeta_{p} d\right) \delta_{r s} \\
& +\frac{i a_{m} a_{p}}{2 \pi} \sin \left(\zeta_{m} d\right) I_{p m q n}\left(k_{l}\right), \\
\Gamma_{r}= & 2 k_{z} a_{p} G_{2}\left(p, k_{x}\right) \exp \left(i k_{x} x_{q}\right),
\end{aligned}
$$

and $r=(q-1) M+p$ and $s=(n-1) M+m$. The integral $I_{p m q n}(k)$ is expressed as

$$
\begin{aligned}
I_{p m q n}\left(k_{l}\right)= & \int_{-\infty}^{\infty} G_{1}(p, \xi) G_{2}(m, \xi)\left(k_{l}^{2}-\xi^{2}\right)^{1 / 2} \\
& \times \exp \left[i \xi\left(x_{n}-x_{q}\right)\right] \mathrm{d} \xi .
\end{aligned}
$$

A technique for efficient evaluation of this integral can be found in Ref. 15 .

\section{B. Far Fields and Transmission Coefficient}

Solution of Eq. (10) allows determination of the unknown coefficients $b_{m}{ }^{n}$ and $c_{m}{ }^{n}$ that can subsequently be used to determine fields by use of Eqs. (3), (5), (7), and (9). For far-zone observations, we can let $x=r_{s}$ $\times \sin \theta_{s}$ and $z-d=r_{s} \cos \theta_{s}$ for $z \geq d$, and $x=r_{t}$ $\times \sin \theta_{t}$ and $z+d=-r_{t} \cos \theta_{t}$ for $z \leq-d$. Then, using the method of steepest descent for $r_{s, t}$ large, we manipulate Eqs. (3) and (5) into the general expression

$$
\begin{aligned}
E_{y}^{s, t}\left(\theta_{s, t}\right)= & \left(\frac{k_{1,3}}{i 2 \pi r_{s, t}}\right)^{1 / 2} \cos \theta_{s, t} \sum_{n=1}^{N} \sum_{m=1}^{M} a_{m}\left(b_{m}{ }^{n} \cos \zeta_{m} d\right. \\
& \left. \pm c_{m}{ }^{n} \sin \zeta_{m} d\right) G_{1}\left(m, k_{s, t}\right) \exp \left[i \left(k_{1,3} r_{s, t}\right.\right. \\
& \left.\left.-k_{s, t} x_{n}\right)\right]
\end{aligned}
$$

where $k_{s}=k_{1} \sin \theta_{s}$ and $k_{t}=k_{3} \sin \theta_{t}$.

The transmission coefficient of the polarizer is defined as the ratio of the total power transmitted by the aperture to that intercepted by the aperture. This corresponds to a detector being placed behind the polarizer such that it captures all the transmitted light. To maintain consistency for comparisons performed below in this paper, the aperture dimension is taken to be $N \Delta x$. Using this convention, we obtain the expression

$$
\begin{aligned}
T= & \frac{1}{N k_{1} \cos \theta} \frac{a}{\Delta x} \frac{\mu_{1}}{\mu_{2}} \sum_{n=1}^{N} \sum_{m=1}^{M} \operatorname{Im}\left[\zeta _ { m } { } ^ { * } \left(b_{m}{ }^{n} \cos \zeta_{m} d\right.\right. \\
& \left.\left.-c_{m}{ }^{n} \sin \zeta_{m} d\right)\left(b_{m}{ }^{n} \sin \zeta_{m} d+c_{m}{ }^{n} \cos \zeta_{m} d\right)^{*}\right] .
\end{aligned}
$$

\section{Infinite Periodic Structure}

In the event that the number of apertures $N$ becomes infinite, the solution methodology can be altered to allow efficient evaluation of the fields. In this case, the fields in regions 1 and 3 are expressed in terms of Floquet harmonics,

$$
\begin{aligned}
E_{y}^{s, t}(x, z)= & \sum_{q=-\infty}^{\infty} \gamma_{q}^{s, t} \exp \left[-i \xi_{q} x \pm i\left(k_{1,3}{ }^{2}-\xi_{q}{ }^{2}\right)^{1 / 2}\right. \\
& \times(z \mp d)], \\
\gamma_{q}^{s, t}= & \frac{1}{\Delta x} \int_{-\Delta x / 2}^{\Delta x / 2} E_{y}^{s, t}(x, \pm d) \exp \left(i \xi_{q} x\right) \mathrm{d} x
\end{aligned}
$$

where $\xi_{q}=k_{x}+2 \pi q / \Delta x$.

With this representation, the problem becomes that of finding the fields in a single aperture $(N=1)$. Enforcement of tangential field continuity leads to the expression

$\gamma_{q}^{s, t}=\frac{1}{\Delta x} \sum_{m=1}^{\infty}\left(b_{m} \cos \zeta_{m} d \pm c_{m} \sin \zeta_{m} d\right) a_{m} G_{2}\left(m, \xi_{q}\right)$,

as well as a matrix equation of the form of Eq. (10) with elements

$$
\begin{aligned}
\Psi_{l B, p m}= & \zeta_{p} a \frac{\mu_{l}}{\mu_{2}} \sin \left(\zeta_{p} d\right) \delta_{p m}+\frac{i a_{m} a_{p}}{\Delta x} \\
& \times \cos \left(\zeta_{m} d\right) F_{p m}\left(k_{l}\right), \\
\Psi_{l C, p m}= & -\zeta_{p} a \frac{\mu_{l}}{\mu_{2}} \cos \left(\zeta_{p} d\right) \delta_{p m} \\
& +\frac{i a_{m} a_{p}}{\Delta x} \sin \left(\zeta_{m} d\right) F_{p m}\left(k_{l}\right),
\end{aligned}
$$

and with $\Gamma_{p}$ given in Eq. (13). It is noteworthy that this matrix equation has a dimension of $M \times M$, in contrast to the $M N \times M N$ equation encountered for the finite-aperture case. The terms $F_{p m}$ are given by the rapidly converging sum

$F_{p m}\left(k_{l}\right)=\sum_{q=-\infty}^{\infty} G_{1}\left(p, \xi_{q}\right) G_{2}\left(m, \xi_{q}\right)\left(k_{l}^{2}-\xi_{q}^{2}\right)^{1 / 2}$.

At this point, we can perform the computation of the transmission coefficient using the expression in Eq. (16) with $N=1$. However, when Eq. (19) is placed into Eq. (17), it should be noted that only a finite number of series terms will be characterized by propagation in the $\pm z$ directions. These terms represent 
plane waves that will propagate to the far zone. For the wire spacings typical in polarizer applications, only the $q=0$ term will propagate to the far field.

\section{Transverse-Magnetic Illumination}

The spectral-domain analysis methodology outlined above can be repeated for TM illumination, with the roles of magnetic and electric fields interchanged. The key difference for the TM case involves an expression for the fields in region 2 because the magnetic field must satisfy proper boundary conditions at the slit sidewalls $\left\{\right.$ i.e., $\partial H_{y} / \partial x=0$ so that $\sin \left[a_{m}\left(x-x_{n}+a\right)\right]$ becomes $\left.\cos \left[a_{m}\left(x-x_{n}+a\right)\right]\right\}$. With this change, the derivation parallels that given above. In the interest of conciseness, the derivation is not repeated here. Details can be found in the references. ${ }^{15}$

\section{E. Computational Considerations}

Although the derivation of the spectral-domain modematching technique is somewhat involved, the resulting algorithm is relatively straightforward to implement. In addition, the method is computationally efficient for typical problems. For example, a representative geometry with a $2 \lambda$ aperture and ten slits (slit width $w=0.12 \lambda$ ) requires only $M=5$ modes for the sums to converge to within four significant digits. Such a computation requires approximately $6 \mathrm{~s}$ on the moderately capable computer used for this study (Hewlett-Packard Visualize B-180L workstation). This is in contrast to numerical methods such as the finite-difference time-domain method in which the simulation requires relatively long run times to characterize the device behavior. It should be mentioned that, in all computations shown in this paper, a convergence test was performed to ensure that enough modes were used to obtain four significant digits of accuracy. Also, as demonstrated in several of the references, the method has been proven to provide accuracy comparable with or better than many techniques such as the method of moments, ${ }^{18}$ Keller's highfrequency diffraction theory, ${ }^{17}$ Kirchhoff's scalar solution, and a rigorous Fourier analysis method for thin structures. ${ }^{15}$ This accuracy coupled with the relative implementation simplicity and speed makes the method highly suitable for the performance of detailed parametric studies of the device behavior.

\section{Results}

In this section we utilize the theory summarized above to investigate the behavior of the wire grid polarizer for different geometric parameters and incident wave characteristics. Throughout the discussion, the following terms are utilized to quantify the device parameters: fill factor, $1-w / \Delta x$; aperture size, $N \Delta x$; slit density (number of slits per wavelength), $\lambda / \Delta x$; and extinction ratio, $T_{\mathrm{TM}} / T_{\mathrm{TE}}$, where the subscripts on the transmission coefficient $T$ denote the polarization of the illumination field.

In much of this study we investigate the behavior of the polarizer transmission coefficient for different geometric configurations. In this exploration, it is important to emphasize that the transmission coefficient is
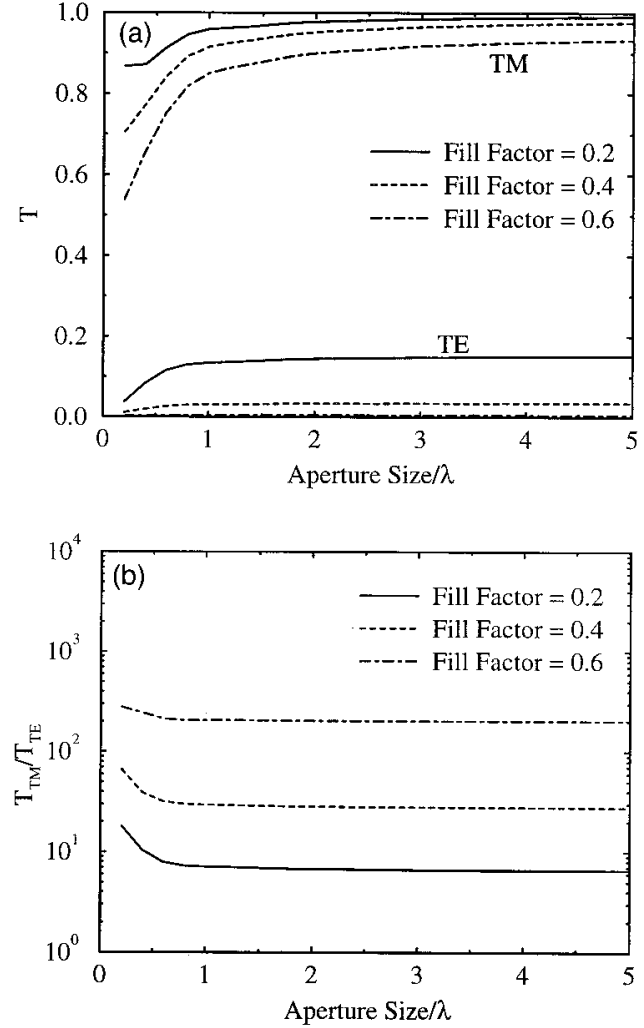

Fig. 2. (a) Transmission coefficient and (b) extinction ratio as a function of aperture size for several values of fill factor $(D=0,5$ slits $(\lambda, M=5$ ).

defined as the ratio of the total power transmitted to the total power intercepted by the polarizer aperture. Unless otherwise indicated, all plots are for normally incident illumination $\left(\theta=0^{\circ}\right)$.

A second important characteristic outlined in the following discussion is the angular distribution of the transmitted energy for the TM polarization. To assess the effect of different geometric parameters on this behavior, the polarizer diffraction pattern is presented as the ratio of the transmitted power density to the power density that would be obtained if the power intercepted by the aperture were radiated isotropically into the space $z<-d$. Mathematically, this is given as

$$
\begin{aligned}
G_{t}\left(\theta_{t}\right) & =10 \log \left[\frac{\eta_{3}\left|H_{y}{ }^{t}\left(\theta_{t}\right)\right|^{2} / 2}{P_{i} / \pi r_{t}}\right], \\
& =10 \log \left[\frac{\eta_{3}}{\eta_{1}} \frac{\mid H_{y}{ }^{t}\left(\left.\theta_{t}\right|^{2}\right.}{\left|H_{i}\right|^{2}} \frac{\pi r_{t}}{N \Delta x \cos \theta}\right],
\end{aligned}
$$

where $P_{i}$ is the power intercepted by the aperture.

\section{A. Effect of Aperture Size}

As a starting point, consider Fig. 2, which shows the transmission behavior as a function of aperture size for several different values of the fill factor for an infinitesimally thin polarizer $(D=0)$. The computations assume 5 slits $/ \lambda(\Delta x=0.2 \lambda)$, and $M=5$ modes to describe the fields within each slit. These plots dem- 
onstrate that, although small apertures tend to have somewhat reduced transmission coefficient values, the transmission and extinction characteristics of apertures with dimensions greater than approximately $1 \lambda$ are relatively insensitive to aperture size. This phenomenon implies that, for larger apertures, the energy contained in the perturbed fields near the aperture edge is relatively small compared with the total energy transmitted. It is noteworthy that the infiniteaperture analysis predicts transmission coefficients of $0.999,0.991$, and 0.953 for TM and 0.162, 0.040, and 0.007 for TE for fill factors of $0.2,0.4$, and 0.6 , respectively. Examination of the curves in Fig. 2(a) reveals that the results from the finite-aperture computation approach these values as the aperture dimension increases. Figure 3, which plots the transmission coefficient versus aperture size for several values of polarizer thickness, shows that the transmission characteristics remain relatively insensitive to aperture dimension even for the finite-thickness case.

Figure 4 shows the diffraction pattern for three different aperture sizes. In contrast to the transmission coefficient, the diffraction pattern is highly sensitive to the aperture dimension. This sensitivity is because the area of the radiating region is directly related to the diffraction pattern beam width.

\section{B. Effect of Wire Properties}

It is important to recognize that fabrication considerations impose constraints on wire grid polarizer design. For example, consider use of microfabrication techniques to create a polarizer for the infrared or visible portion of the spectrum. .,20,21 $^{\text {Minimum fea- }}$ ture size limitations determine the slit density, fill factor, and wire aspect ratio, and it is therefore crucial to understand the impact of these parameters on polarizer transmission behavior.

To begin our examination of the effect of wire characteristics on the polarizer behavior, we reconsider the data in Fig. 2. These results clearly indicate that, as the fill factor is increased, the transmission coefficient for both polarizations decreases because of the increased area occupied by the conducting wire. How-

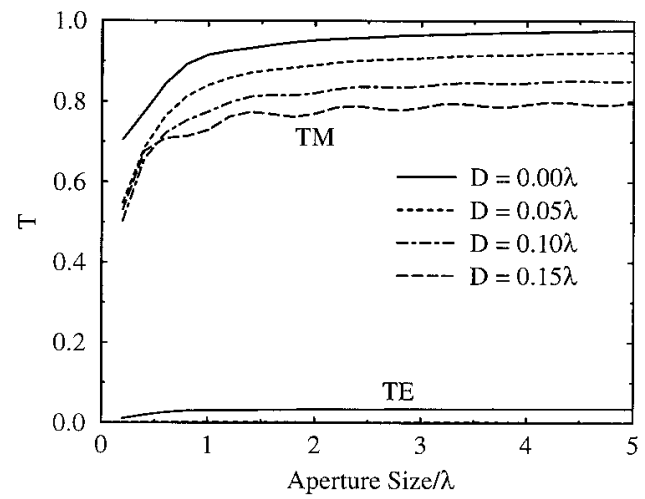

Fig. 3. Transmission coefficient as a function of aperture size for several values of polarizer thickness (fill factor is $0.4,5 \mathrm{slits} / \lambda, M=$ $5)$.

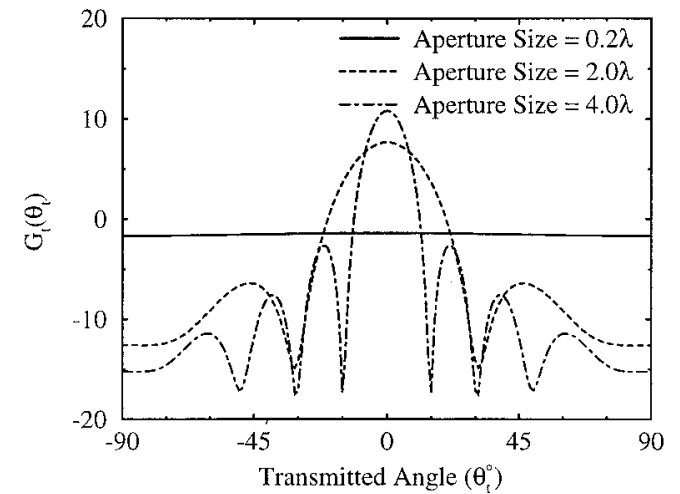

Fig. 4. Transmitted diffraction pattern for a polarizer with three different aperture sizes $(D=0,5$ slits $/ \lambda$, fill factor is $0.4, M=5)$.

ever, because the TE polarization is more strongly impacted by the increase in conductor size, the extinction ratio also increases with fill factor. ${ }^{20}$ Figure 5 depicts the polarizer transmission behavior as a function of the slit density and fill factor for an infinitesimally thin polarizer. These results illustrate that the above-observed fill factor trends remain the same as the slit density is varied. These plots also demonstrate a substantial increase in extinction ratio as the slit density is increased. This behavior is consistent with expectation because an increase in slit density implies an increased number of thinner, more closely spaced wires. Such a distribution of the conductor in the aperture tends to be less disruptive to the TM polarization and more disruptive to the TE polarization.

Figure 3 illustrates a slight decrease in transmission coefficient as the thickness of the wires increases. This behavior is more dramatically demonstrated in Fig. 6, which shows the transmission behavior as a function of fill factor and polarizer thickness. It is noteworthy that, despite the drop in power transmission, the extinction ratio increases sharply with increasing fill factor, particularly for thicker wires. This latter effect can be explained when we consider that the narrow, finite-thickness slit represents a parallel-plate waveguide that supports only the lowest-order transverse-electromagnetic (TEM) mode. Because the TM wave is properly polarized to excite this TEM mode, it passes through relatively easily with reflections that are due mainly to the impedance mismatch between the incident plane wave and the propagating mode. However, the TE waveguide modes excited by the incident TE plane wave will be cut off, and therefore TE transmission falls off dramatically with $D$.

Figure 7 illustrates the effect of polarizer thickness and fill factor on the radiation characteristics. Clearly, both parameters exercise a marginal influence on the pattern shape, with the most notable effects being present in the wide angular regions where the transmitted power concentration is small. Naturally, at a high fill factor with thick wires, the pattern peak is noticeably reduced because of the decreased transmission power. 


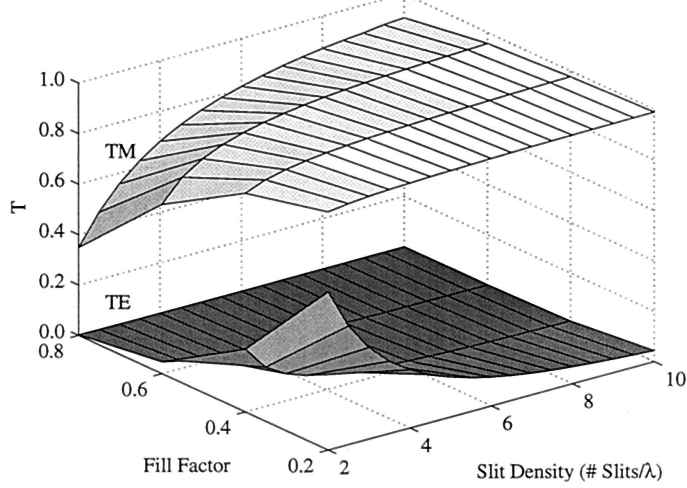

(a)

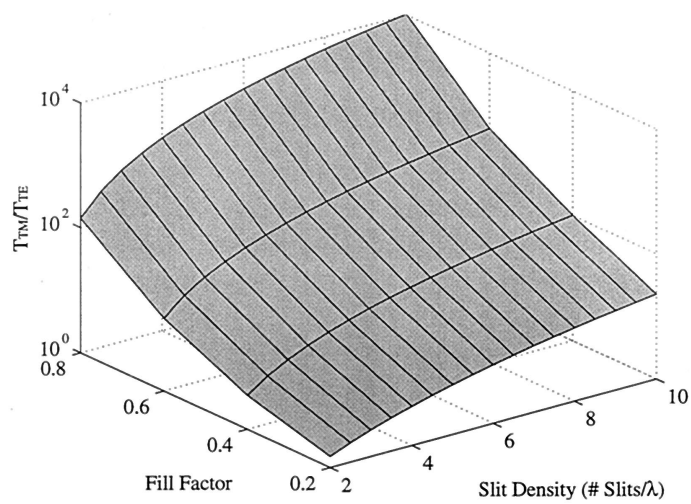

(b)

Fig. 5. (a) Transmission coefficient and (b) extinction ratio as a function of slit density and fill factor $(D=0$, aperture size is $2 \lambda$, $M=10)$.

\section{Effect of Material Properties}

One key strength of the analysis methodology chosen is its ability to assess the role of material properties on the polarizer performance. Figure 8 shows the transmission characteristics as a function of the relative permittivity $\epsilon_{r 1}$ for region 1 for four different values of the relative permittivity in regions 2 and $3\left(\epsilon_{r 2}=\epsilon_{r 3}\right)$. Naturally, the transmission power observed in these plots is related to the Fresnel transmission coefficient that would occur at an interface between two dissimilar materials with no polarizer metal. However, examination of this Fresnel power transmissivity, expressed as

$$
T_{F}=1-\left|\frac{\epsilon_{r 1} / \epsilon_{r 2}-1}{\epsilon_{r 1} / \epsilon_{r 2}+1}\right|^{2},
$$

shows that this behavior alone would indicate perfect transmission when the two media are the same and a monotonic decrease in $T_{F}$ as $\epsilon_{r 1}$ varies from this equality value. However, as the value of permittivity (particularly that in region 2) increases, the wire electrical spacing also increases, implying the possibility of increased power transmission. Therefore the trends observed in Fig. 8 clearly represent an interesting in-

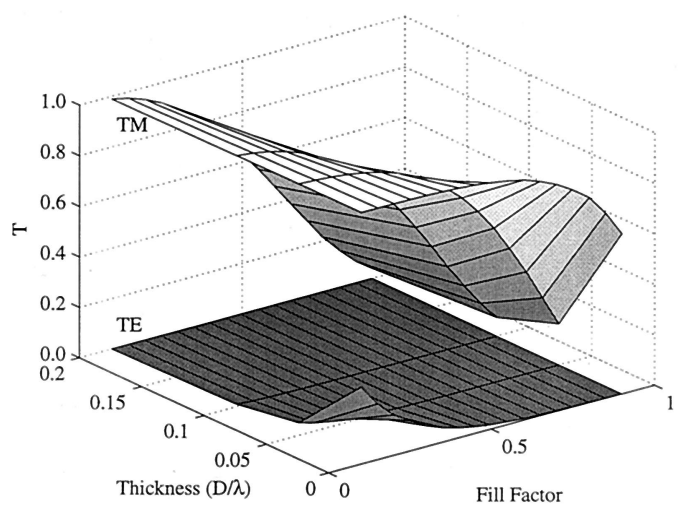

(a)

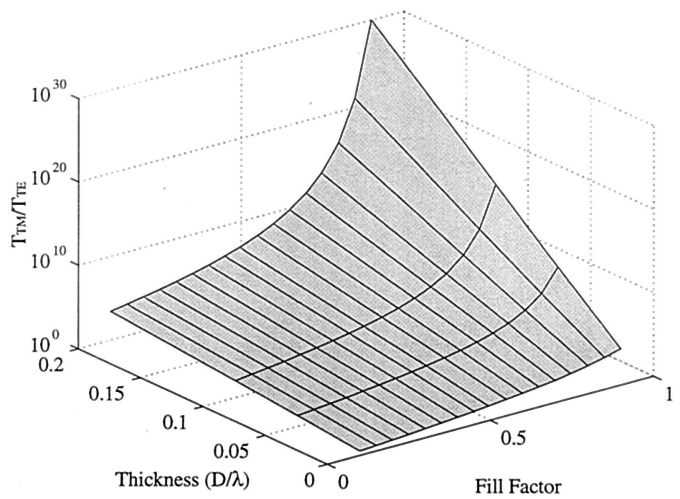

(b)

Fig. 6. (a) Transmission coefficient and (b) extinction ratio as a function of fill factor and polarizer thickness (aperture size is infinite, 5 slits $/ \lambda, M=5$ ).

terplay between Fresnel transmission and polarizer electrical properties. It is intriguing, however, that the extinction ratio has a well-structured behavior as a function of material dielectric constants.

Figure 9 illustrates the diffraction pattern for different combinations of material relative permittivity.

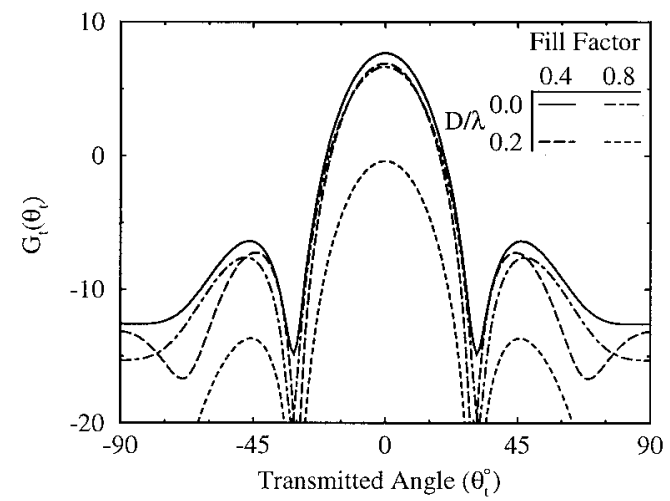

Fig. 7. Transmitted diffraction pattern for a polarizer with three different values of thickness (aperture size is $2 \lambda, 5 \mathrm{slits} / \lambda$, fill factor is $0.4, M=5$ ). 

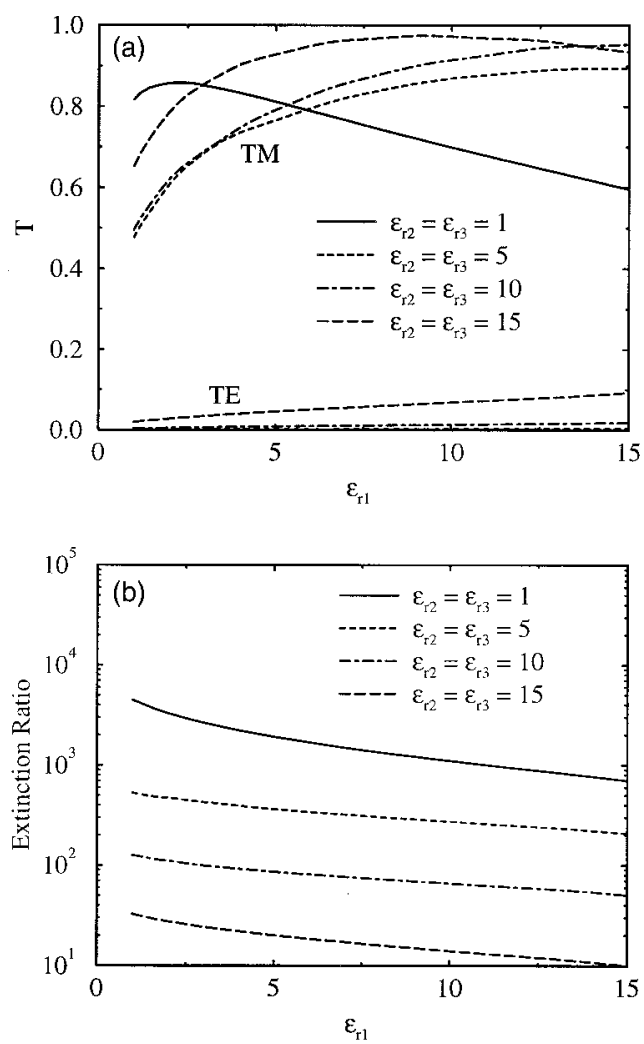

Fig. 8. (a) Transmission coefficient and (b) extinction ratio as a function of relative permittivity of region 1 for several values of relative permittivity of regions 2 and $3(D=0.1 \lambda$, fill factor is 0.4 , aperture size is $2 \lambda, 5 \operatorname{slits} / \lambda, M=5$ ).

As can be seen, the pattern shape is essentially controlled by the material properties at the polarizer output. This observation can be explained when we realize that the material properties in region 3 control the electrical size of the aperture and therefore the angular distribution of the transmitted energy.

Although the analysis method utilized is not able to characterize the effect of imperfectly conducting wires, past research by use of more advanced computational models ${ }^{14}$ has demonstrated that, as long as such materials have relatively high conductivity, the transmis-

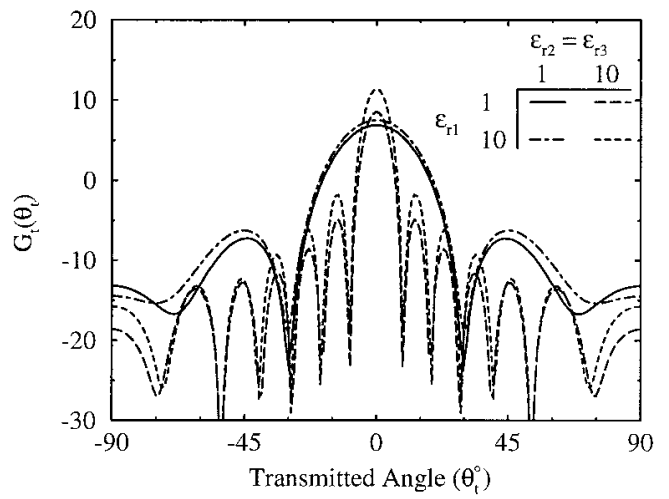

Fig. 9. Transmitted diffraction pattern for a polarizer with different material permittivities $(D=0.2 \lambda$, aperture size is $2 \lambda, 5$ slits/ $\lambda$, fill factor is $0.4, M=5$ ). sion of the TM wave remains relatively unchanged. However, because imperfect conductors allow increased transmission of the TE wave, the extinction ratio tends to be reduced by approximately an order of magnitude. It is important to include such information in the design of practical polarizers.

\section{Effect of Incidence Angle}

Finally, we consider the polarizer transmission properties as a function of the incidence angle. Figure 10 shows the effect of incidence angle for several different aperture sizes. Because of the nonzero grating thickness $(D=0.1 \lambda)$, the TE transmission power is small for all angles and apertures. It should be emphasized that the transmission coefficient was normalized by $\cos \theta$ to accurately show the behavior near $\theta=90^{\circ}$ where the denominator of $T$ goes to zero. The fact that the finite-sized apertures have a nonzero normalized transmission coefficient for the TM polarization at $\theta=90^{\circ}$ implies that the polarizer structure perturbs the incident field and produces transmitted power even when no power is normally incident on the structure. However, little power is transmitted for the TE polarization in this case, resulting in the large extinction ratio observed.

Figure 11 illustrates the diffraction pattern for different arrival angles of the incident plane wave. These curves reveal the expected shift in the main beam direction to match that of the incident wave as
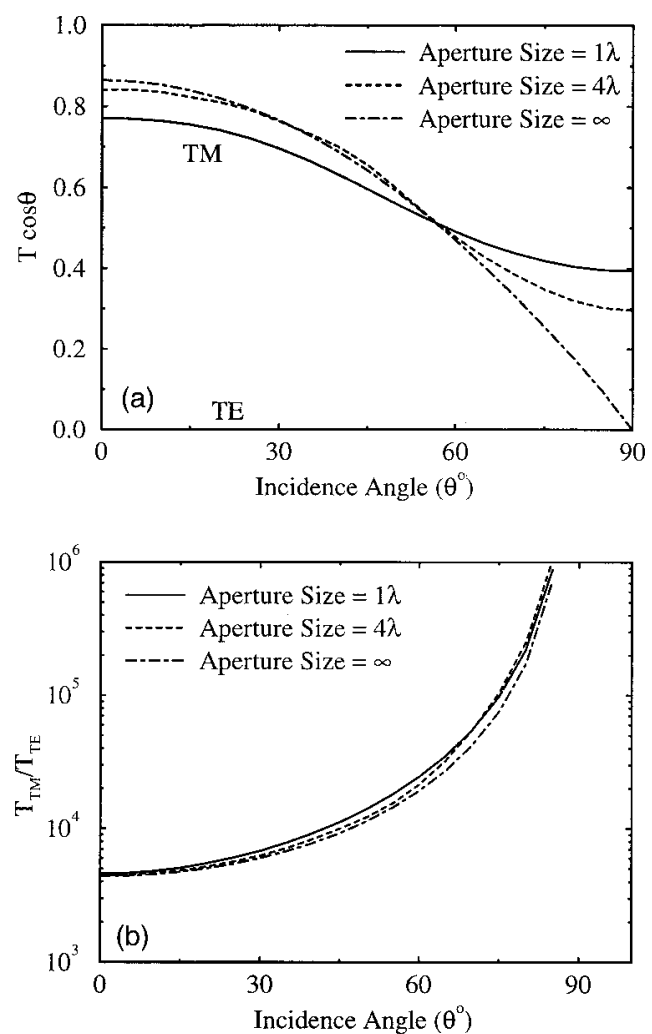

Fig. 10. (a) Normalized transmission coefficient and (b) extinction ratio as a function of plane-wave incidence angle for several aperture sizes $(D=0.1 \lambda$, fill factor is $0.4,5$ slits $/ \lambda, M=5)$. 


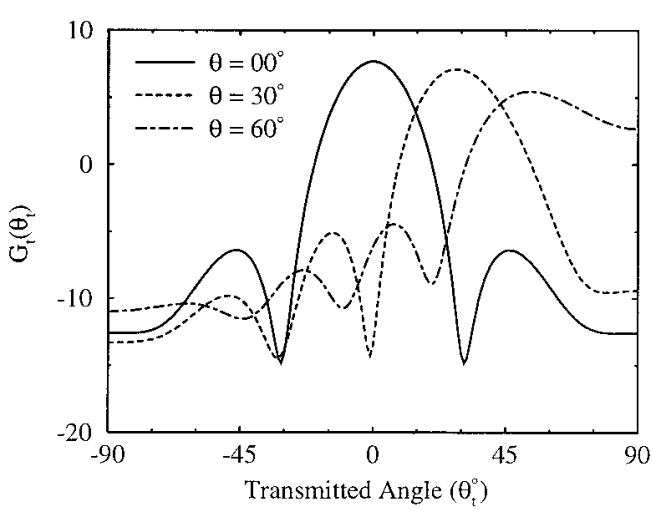

Fig. 11. Transmitted diffraction pattern for a polarizer with three different plane-wave incidence angles $(D=0$, aperture size is $2 \lambda$, 5 slits $/ \lambda$, fill factor is $0.4, M=5$ ).

well as a decrease in power in the main radiation lobe and an increase in the sidelobes.

\section{Summary}

In this paper we have provided a detailed examination of the transmission characteristics of perfectly conducting, finite-aperture wire grid polarizers. The analysis approach consisted of a rigorous modematching solution coupled with a spectral representation of the scattered fields. An extension to this theory that incorporated Floquet analysis allowed simulation of infinitely large, periodic polarizers as well. This analysis methodology was used to investigate the transmission coefficient for both TM and TE polarizations, the extinction ratio, and the diffraction pattern for a wide variety of geometric configurations and incident field characteristics. These studies reveal that, for apertures larger than approximately one wavelength, the transmission coefficient and extinction characteristics are largely insensitive to aperture dimension. Also demonstrated was a decrease in the transmission coefficient for both polarizations and an increase in the extinction ratio with increasing fill factor or increasing thickness.

Many applications require both a large extinction ratio and a high optical throughput. On the basis of the above results, the extinction ratio can, in general, be augmented when the slit density, fill factor, or thickness or some combination of these parameters is increased. However, a high optical throughput is achieved with a low fill factor, or, if the fill factor is constrained to be large, by a decrease in the polarizer thickness. Clearly, the design of a wire grid polarizer for a particular application involves distinct trade-offs between the extinction ratio and the optical throughput.

The research of G. P. Nordin was supported by National Science Foundation CAREER Award ECS9625040 .

\section{References}

1. G. R. Bird and M. Parrish, Jr., "The wire grid as a nearinfrared polarizer," J. Opt. Soc. Am. 50, 886-891 (1960).

2. J. B. Young, H. A. Graham, and E. W. Peterson, "Wire grid infrared polarizer," Appl. Opt. 4, 1023-1026 (1965).

3. M. G. Moharam and T. K. Gaylord, "Rigorous coupled-wave analysis of metallic surface-relief gratings," J. Opt. Soc. Am. A 3, 1780-1787 (1986).

4. M. G. Moharam, E. B. Grann, D. A. Pommet, and T. K. Gaylord, "Formulation for stable and efficient implementation of the rigorous coupled-wave analysis of binary gratings," J. Opt. Soc. Am. A 12, 1068-1076 (1995).

5. E. Chen and S. Y. Chou, "A novel device for detecting the polarization direction of linear polarized light using integrated subwavelength gratings and photodetectors," IEEE Photon. Technol. Lett. 9, 1259-1261 (1997).

6. J. Guo and D. J. Brady, "Fabrication of high-resolution micropolarizer arrays," Opt. Eng. 36, 2268-2271 (1997).

7. G. P. Nordin, J. T. Meier, P. C. Deguzman, and M. W. Jones, "Micropolarizer array for infrared imaging polarimetry," J. Opt. Soc. Am. A 16, 1184-1193 (1999).

8. M. Born and E. Wolf, Principles of Optics, 5th ed. (Pergamon, Tarrytown, N.Y., 1975).

9. K. Hirayama, E. N. Glytsis, and T. K. Gaylord, "Rigorous electromagnetic analysis of diffraction by finite-number-of-periods gratings," J. Opt. Soc. Am. A 14, 907-917 (1997).

10. D. W. Prather, M. S. Mirotznik, and J. N. Mait, "Boundary integral methods applied to the analysis of diffractive optical elements," J. Opt. Soc. Am. A 14, 34-43 (1997).

11. D. W. Prather, J. N. Mait, M. S. Mirotznik, and J. P. Collins, "Vector-based synthesis of finite aperiodic subwavelength diffractive optical elements," J. Opt. Soc. Am. A 15, 1599-1607 (1998).

12. A. Wang and A. Prata, Jr., "Lenslet analysis by rigorous vector diffraction theory," J. Opt. Soc. Am. A 12, 1161-1169 (1995).

13. M. Schmitz and O. Bryngdahl, "Rigorous concept for the design of diffractive microlenses with high numerical apertures," J. Opt. Soc. Am. A 14, 901-906 (1997).

14. M. A. Jensen and G. P. Nordin, "Finite-aperture wire grid polarizers," J. Opt. Soc. Am. A 17, 2191-2198 (2000).

15. Y. S. Kim, H. J. Eom, J. W. Lee, and K. Yoshitomi, "Scattering from multiple slits in a thick conducting plane," Radio Sci. 30, 1341-1347 (1995).

16. O. M. Mendez, M. Cahilhac, and R. Petit, "Diffraction of a twodimensional electromagnetic beam wave by a thick slit pierced in a perfectly conducting screen," J. Opt. Soc. Am. 73, 328-331 (1983).

17. S. H. Kang, H. J. Eom, and T. J. Park, "TM-scattering from a slit in a thick conducting screen: revisited," IEEE Trans. Microwave Theory Tech. 41, 895-899 (1993).

18. T. J. Park, S. H. Kang, and H. J. Eom, "TE-scattering from a slit in a thick conducting screen: revisited," IEEE Trans. Antennas Propag. 42, 112-114 (1994).

19. Y.-K. Kok, "General solution to the multiple-metallic-grooves scattering problem: the fast-polarization case," Appl. Opt. 32, 2573-2581 (1993).

20. B. Stenkamp, M. Abraham, W. Ehrfeld, E. Knapek, M. Hintermaier, M. T. Gale, and R. Morf, "Grid polarizer for the visible spectral region," in Nanofabrication Technologies and Device Integration, W. Karthe, ed., Proc. SPIE 2213, 288-296 (1994).

21. H. Lochbihler and R. Depine, "Diffraction from highly conducting wire gratings of arbitrary cross-section," J. Mod. Opt. 40, 1273-1298 (1993). 